\title{
Biomechanical comparison of three stand-alone lumbar cages - a three-dimensional finite element analysis
}

\author{
Shih-Hao Chen ${ }^{1}$, Ming-Chieh Chiang ${ }^{2}$, Jin-Fu Lin ${ }^{3}$, Shang-Chih Lin ${ }^{4}$ and Ching-Hua Hung ${ }^{2 *}$
}

\begin{abstract}
Background: For anterior lumbar interbody fusion (ALIF), stand-alone cages can be supplemented with vertebral plate, locking screws, or threaded cylinder to avoid the use of posterior fixation. Intuitively, the plate, screw, and cylinder aim to be embedded into the vertebral bodies to effectively immobilize the cage itself. The kinematic and mechanical effects of these integrated components on the lumbar construct have not been extensively studied. A nonlinearly lumbar finite-element model was developed and validated to investigate the biomechanical differences between three stand-alone (Latero, SynFix, and Stabilis) and SynCage-Open plus transpedicular fixation. All four cages were instrumented at the L3-4 level.
\end{abstract}

Methods: The lumbar models were subjected to the follower load along the lumbar column and the moment at the lumbar top to produce flexion (FL), extension (EX), left/right lateral bending (LLB, RLB), and left/right axial rotation (LAR, RAR). A $10 \mathrm{Nm}$ moment was applied to obtain the six physiological motions in all models. The comparison indices included disc range of motion (ROM), facet contact force, and stresses of the annulus and implants.

Results: At the surgical level, the SynCage-open model supplemented with transpedicular fixation decreased ROM (>76\%) greatly; while the SynFix model decreased ROM 56-72\%, the Latero model decreased ROM 36-91\%, in all motions as compared with the INT model. However, the Stabilis model decreased ROM slightly in extension (11\%), lateral bending (21\%), and axial rotation (34\%). At the adjacent levels, there were no obvious differences in ROM and annulus stress among all instrumented models.

Conclusions: ALIF instrumentation with the Latero or SynFix cage provides an acceptable stability for clinical use without the requirement of additional posterior fixation. However, the Stabilis cage is not favored in extension and lateral bending because of insufficient stabilization.

Keywords: ALIF, Anterior lumbar interbody fusion, Stand-alone cage, Finite element analysis

\section{Background}

The lumbar interbody cage is an improvement in spinal fusion which facilitates stabilization of the motion segments and relieves discogenic back pain [1,2]. The common design is either cylindrical or trapezoid in shape and often uses serrated anchorages on the upper and lower surfaces to prevent loosening or subsidence of the cage [3-6]. Stand-alone cages have been used in ALIF

\footnotetext{
* Correspondence: chhung@mail.nctu.edu.tw

${ }^{2}$ Department of Mechanical Engineering, National Chiao Tung University,

1001 University Road, Hsinchu 30010, Taiwan

Full list of author information is available at the end of the article
}

treatment and their ability to stabilize the intervertebral motion has been reported to be superior in flexion and bending to extension and rotation $[7,8]$. In clinical use, the construct stability can be further enhanced by the supplementation of posterior fixation such as pedicle or facet screws $[9,10]$. However, the significant morbidities of the combined anterior and posterior approaches have been mentioned [11]. These drawbacks may be overcome through the use of newly designed ALIF cages that can be inserted via a single anterior or lateral approach with minimal operative morbidity and without causing damage to posterior bony elements and neural, vascular,

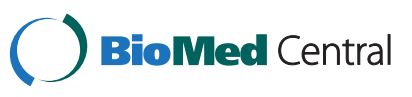




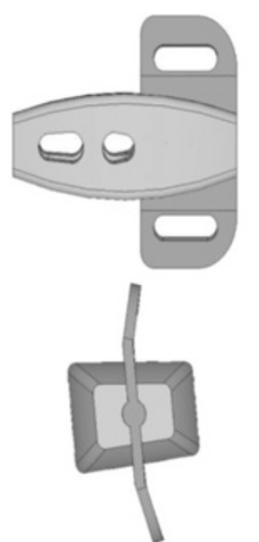

(A)

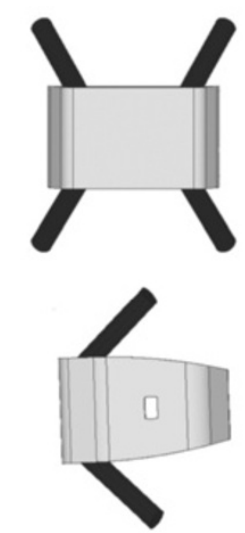

(B)
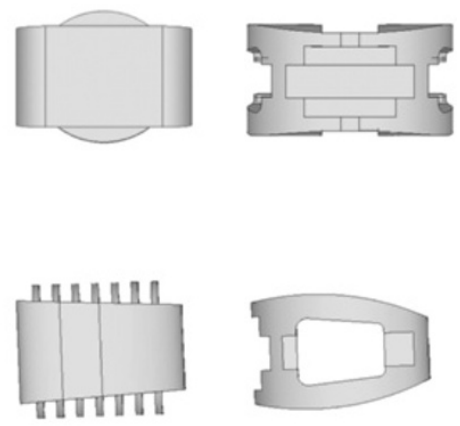

(C)
(D)

Figure 1 Front and side views of the four ALIF cages were used in this study. (A) Latero. (B) SynFix. (C) Stabilis. (D) SynCage-Open.

and muscular tissues [12]. In the past, three stand-alone ALIF cages have been developed, consisting of a trapezoid frame that incorporates the anteriorly stabilizing components (Figure 1). The Latero system (Latero; A-Spine Asia, Taipei, Taiwan) integrates a lateral vertebral plate into the trapezoid frame which is bent to be parallel to the coronal plane. The SynFix system (SynFix; Synthes Spine Inc., PA, USA) uses four screws to lock the adjacent vertebrae. The Stabilis system (Stabilis; Stryker, Michigan, USA) accommodates a threaded cylinder to anchor the superior and inferior endplates. The stabilizing mechanisms of the Latero plate, SynFix screws, and Stabilis cylinder use the vertebrae as fulcrums to immobilize the bone-cage construct $[12,13]$.

In the literature, comparisons between the different stand-alone ALIF cages have been extensively conducted by the experimental, numerical, and clinical methods $[1,2,7]$. Using human cadavers as specimens, the threedimensional stiffness tests in Schleicher's study [1] demonstrated the effective stabilization ability of the stand-alone SynFix cage in all motion directions. In a previous study by the current authors [14] the numerical results showed no differences of ROM in extension and lateral bending between the Stabilis and SynCage-Open (Synthes Spine, Inc., PA, USA). Except for the differences among cage frames, the stabilizing mechanism might contribute to the postoperative outcome of the stand-alone ALIF [12]. From the biomechanical viewpoint, however, the insertion depth and holding power were quite different between the plate, screw, and cylinder, thus, potentially affecting the stabilizing ability of the stabilizing mechanisms [15].

After surgery, both initial stability and loading transmission of the ALIF level play essential roles in the fusion rate of the instrumented region and the junctional problem of the adjacent region [16]. Especially for osteoporotic bones, bone-cage loosening might result in cage subsidence, interfacial migration, and subsequent nonunion with loss of disc height [9,15]. Jost et al. [15] experimentally measured the interfacial strengths of three different bone-cage constructs and declared no significant differences between the threaded Ray cage, the rectangular Brantigan cage, and the porous Contact $\mathrm{Fu}$ sion cage. Cho et al. [2] demonstrated that the standalone ALIF cage could assure good clinical results in the surgical treatment of symptomatic lumbar intervertebral foraminal stenosis in a mid-term follow up. To the best of the authors' knowledge, little study has been dedicated to the detailed investigation of Stabilis performance to date. Moreover, there has been no extensive study devoted to investigate the kinematic and mechanical differences between the plate-, screw-, and threadtype ALIF cages. This constituted the motive of the current study.

Both stress distribution and interfacial micromotion of the bone-cage construct are not easily detectable by experimental methods [17-21]. This study used the finiteelement method to evaluate the biomechanical effects of the stand-alone ALIF cages on the kinematic and mechanical behaviors of the adjacent tissues and cages. Three stand-alone ALIF cages (Latero, SynFix, and Stabilis) and one traditional ALIF cage (SynCage-Open) with transpedicular fixation were instrumented into the lumbar models and compared. The detailed investigation focused on the stress distribution and stabilization ability of the Latero plate, SynFix screws, and Stabilis threads. An intact model was used as the comparison baseline and the vertebral strengths were systematically varied. The outcome of this study provides insight into the biomechanical properties of the bone-cage stabilizing mechanism within normal and osteoporotic bones. 


\section{Methods}

One intact and four instrumented models of the lumbar spine were constructed in this study. The first was the intact model (INT model) to serve as the comparison baseline. The other four models were instrumented with ALIF cages (Latero, SynFix, Stabilis, and SynCageOpen) and/or transpedicular fixation at the L3-4 level (Figures 1c-d).

\section{Intact models}

A three-dimensional nonlinear model of the lumbar spine was constructed from L1 to L5 levels (Figure 2). The lumbar geometry was reconstructed from 1-mm computed tomography scans of a middle-aged male. The CT-scanning images of only one vertebra were used to build the entire lumbar column. The other vertebrae were duplicated and spanned by the intervertebral discs that were manually developed by the CAD software. This makes the vertebral bodies, posterior elements, and associated processes quite similar in shape and size. The pre-procedures of the lumbar FE model were established using ANSYS, Ed.9.0 software (ANSYS Inc., Canonsburg, PA, USA). The INT model was an osseo-ligamentous lumbar spine which includes the vertebrae, intervertebral discs, endplates, posterior elements, and all seven ligaments. The eight-node solid elements were used for modeling the cortical bone, cancellous bone, posterior element, and endplate. The material properties of all tissues were assumed to be homogeneous and transversely isotropic [22]. The intervertebral disc consisted of annulus ground substance, nucleus pulpous, and collagen fibers embedded in the ground substance. The nonlinear annulus ground substance was simulated by using the hyper-elastic Mooney-Rivlin formulation [23]. In the radial direction, twelve double cross-linked fiber layers were defined to decrease elastic strength proportionally from the outermost layer to the innermost. The collagen fibers in different annulus layers were strengthened by the weight factor using the approach from a previous study by the current authors [20]. The weight factors of the elastic modulus were 1.0 at outermost layers, $1-3,0.9$ at layers $4-6,0.75$ at layers $7-9$, and 0.65 at the innermost layers, 10-12. The nucleus pulpous was modeled as an incompressible fluid with a bulk modulus of $1666.7 \mathrm{MPa}$ by eight-node fluid elements [22].

No morphological change in vertebrae was simulated in this study. Only decrease in vertebral strength was assumed to model the biomechanical property of the osteoporotic bone. There were two vertebral strengths simulated in this study: normal and osteoporotic models. Compared to the normal bone, "osteoporosis" was defined as a decrease of $66 \%$ in the elastic modulus for cancellous bone and a decrease of $33 \%$ for the cortical bone [24]. All seven ligaments and collagen fibers were simulated by two-node bilinear link elements with uniaxial tension resistance only, which were arranged in an anatomically correct direction [25]. The cross-sectional areas of each ligament and material properties of the spine were obtained from previous studies (Table 1) [20,22,23,25]. The facet joint was modeled with sliding and nonpenetrating behavior using an eight-node surface-to

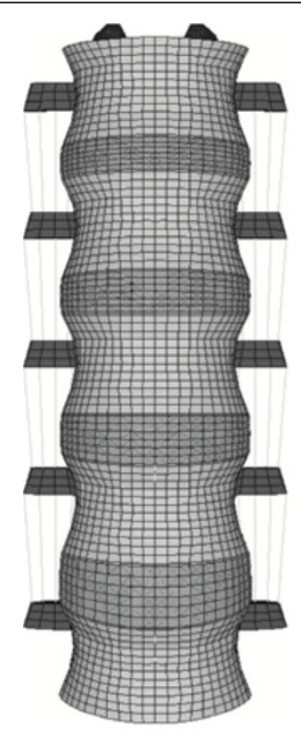

(A)

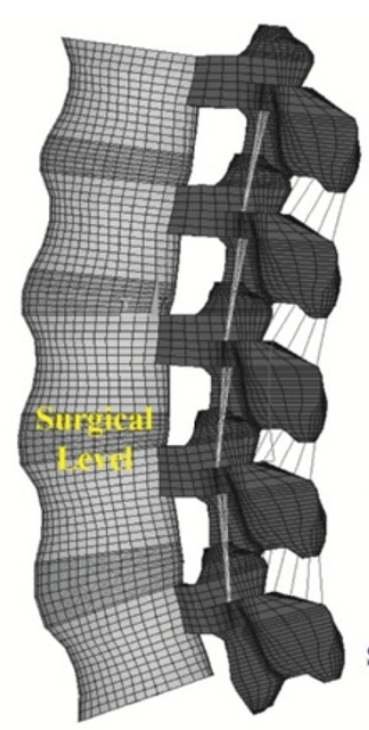

(B)

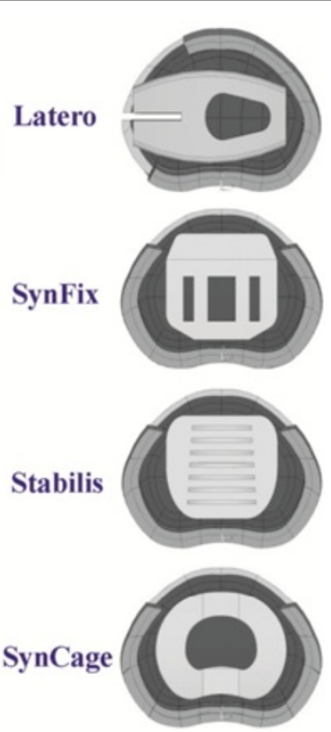

(C)

Figure 2 The lumbar finite-element model used in this study. (A) Intact model from L1 to L5 levels. (B) Instrumented model at the L3-4 level. (C) Four ALIF cages instrumented at the L3-4 level. 
Table 1 Material properties used in the FE model

\begin{tabular}{|c|c|c|c|c|c|}
\hline \multirow[t]{2}{*}{ Material } & \multirow[t]{2}{*}{ Element Type } & \multirow{2}{*}{\multicolumn{2}{|c|}{$\begin{array}{c}\text { Young's Modulus (MPa) } \\
\text { Poisson's Ratio }\end{array}$}} & \multirow{3}{*}{$\begin{array}{l}\text { Area } \\
\left(\mathrm{mm}^{2}\right)\end{array}$} & \multirow[t]{2}{*}{ Reference } \\
\hline & & & & & \\
\hline Bone & & $E x=11300$ & $v x y=0.484$ & & {$[22]$} \\
\hline \multirow[t]{5}{*}{ Cortical } & 8-node SOLID185 & $E y=11300$ & $v x z=0.203$ & & \\
\hline & & $E z=22000$ & $v y z=0.203$ & & \\
\hline & & $G x=3800$ & & & \\
\hline & & $\mathrm{Gy}=5400$ & & & \\
\hline & & $\mathrm{Gz}=5400$ & & & \\
\hline \multirow[t]{6}{*}{ Cancellous } & 8-node SOLID185 & $E x=140$ & $v x y=0.45$ & & [22] \\
\hline & & Ey $=140$ & $v x z=0.315$ & & \\
\hline & & $E z=200$ & $r y z=0.315$ & & \\
\hline & & $G x=48.3$ & & & \\
\hline & & $G y=48.3$ & & & \\
\hline & & $\mathrm{Gz}=48.3$ & & & \\
\hline Posterior bone & 8-node SOLID185 & 3500 & 0.25 & - & {$[22]$} \\
\hline Disc & & & & & [22] \\
\hline Nucleus pulposus & 8-node FLUID80 & 1666.7 & - & - & [22] \\
\hline \multirow[t]{2}{*}{ Ground Substance } & 8-node SOLID185 & $C_{10}=0.42$ & - & - & \\
\hline & & $C_{01}=0.105$ & & & \\
\hline Annulus fibers & 2-node LINK10 & & & & [23] \\
\hline Outermost & & 550 & - & 0.76 & \\
\hline Second & & 495 & - & 0.5923 & \\
\hline Third & & 412.5 & - & 0.4712 & \\
\hline Innermost & & 357.5 & - & 0.3572 & \\
\hline Cartilaginous Endplates & 8-node SOLID185 & 24 & 0.4 & - & {$[22]$} \\
\hline Ligaments & 2-node LINK10 & & & & {$[25]$} \\
\hline ALL & & 7.8 & - & 24 & \\
\hline PLL & & 10 & - & 14.4 & \\
\hline $\mathrm{TL}$ & & 10 & - & 3.6 & \\
\hline LF & & 15 & - & 40 & \\
\hline ISL & & 10 & - & 26 & \\
\hline SSL & & 8 & - & 23 & \\
\hline $\mathrm{CL}$ & & 7.5 & - & 30 & \\
\hline Latero (PEEK) & 8-node SOLID185 & 6500 & 0.2 & & \\
\hline Latero-plate (Titanium alloy) & 8-node SOLID185 & 110000 & 0.3 & & \\
\hline SynFix-LR (PEEK) & 8-node SOLID185 & 6500 & 0.2 & & \\
\hline Locking screw (Titanium alloy) & 8-node SOLID185 & 110000 & 0.3 & & \\
\hline Stabilis (Titanium alloy) & 8-node SOLID185 & 110000 & 0.3 & & \\
\hline SynCage-Open (Titanium alloy) & 8-node SOLID185 & 110000 & 0.3 & - & \\
\hline Pedicle screw (Titanium alloy) & 8-node SOLID185 & 110000 & 0.3 & & \\
\hline
\end{tabular}

ALL anterior longitudinal ligament; $P L L$ posterior longitudinal ligament; $T L$ transverse ligament; $L F$ ligamentum flavum; ISL interspinous ligament; SSL supraspinous ligament; $C L$ capsular ligament.

-surface contact element which can slide between threedimensional target surfaces. The initial gap between a paired facet was kept within $0.5 \mathrm{~mm}$ and the coefficient of friction was set at $0.1[20,22]$.

\section{ALIF models}

The Latero cage can be interlocked with a bent lateral plate that is inserted parallel to the coronal plane and into the vertebral bodies, thus preventing cage loosening. 
The SynFix cage consists of a trapezoid frame incorporated with an anterior plate and screws to stabilize the cage body. The Stabilis cage is a trapezoid frame with a threaded cylinder at the middle region to increase the bone-purchasing ability. Without the serrated anchorages, the final ALIF model of the SynCage-Open cage is further instrumented with bilateral pedicle screws to enhance the construct stability. The models of all cage systems were established by SolidWorks, Ed. 2012 software (SolidWorks Corporation, Concord, MA, USA). The spikes of the Latero cage and the screw threads of the SynFix cage were omitted to simplify numerical calculation (Figure 1).

For ALIF simulation, the L3-4 level of the INT model underwent partial discectomy and total nuclectomy. For the SynFix, Stabilis and SynCage-Open models, the anterior approach was used to remove the anterior longitudinal ligament, anterior and a half inner layer of the annulus, and the entire nucleus pulpous. For the Latero model, the lateral approach was adapted to remove the lateral and a half inner layer of the annulus and the entire nucleus pulpous. All the other ligaments of the three models were preserved.

Accordingly, the Latero, SynFix, Stabilis, and SynCageOpen peek cages were modeled and inserted into the L3-4 level. The friction coefficient of the bone-cage interfaces was 0.8 to mimic a serrated surface for the initial stability of the trapezoid frame [14]. The SynCage-Open model was supplemented with bilateral transpedicular fixation. In the SynCage-Open and SynFix models, the pedicle screw (6-mm diameter) and locking screw (4-mm diameter) were respectively modeled with three-dimensional beam elements. The bone-screw interfaces were assumed to be fully bonded to simulate intimate bone-screw purchase. The numbers of elements and nodes were 98,988/135,786, $112,087 / 253,492,114,849 / 162,169$, and 106,436/180,875 for the Latero, SynFix, Stabilis, and SynCage-Open models, respectively. The material properties of the ALIF cages, supplementary components, and transpedicular fixator were listed in Table 1.

\section{Finite-element analyses}

Only vertical compression onto the lumbar top can potentially lead to excessive movement of the lumbar column [16]. In this study, the follower load was used to constrain each motion segment of the lumbar model with the two-node truss elements that induce contractions for a given temperature change [26]. In principle, the ideal follower load remains tangent to the spine curve, and each spinal segment is loaded in nearly pure compression without artifact motions. Using the trialand-error method, the attached points of truss elements were modified to optimize the follower load path approximated through the instantaneous center of rotation at each motion segment [27]. This study cautiously decreased the temperature of the truss elements to produce a 400-N follower load for minimizing the ROM of each motion segment $\left(<0.2^{\circ}\right)$. Subsequently, a $10-\mathrm{Nm}$ moment was applied to the lumbar top to simulate flexionextension, left/right lateral bending, and left/right axial rotation, respectively. During simulation, the bottom of the L5 vertebral body was fixed completely. The comparison indices were intersegmental ROM, facet force, and stresses of annulus, implant, and endplate. The annulus stress is the stress of the ground substance. From the biomechanical viewpoint, the annulus fibers are mainly responsible for the tension. However, the ground substance can bear the various loads that always exist within the intervertebral discs. Previous in vitro studies demonstrated that a disc may prolapse under certain load combinations of flexion, lateral bending, axial rotation, and axial compression [28-30]. Only the stress of the annulus fibers cannot provide the sufficient information about the disc loads. Consequently, this study uses the stress of the ground substance as the index of the disc prolapse and herniation.

\section{Results}

This study used four parameters as the comparison indices, including intersegmental ROMs, annulus stress, endplate stress, and facet contact forces (Figures 3, 4, 5, 6, 7 and 8). There were five models: one intact (INT), 3 stand-alone ALIF cages (Latero, SynFix, and Stabilis), and one established fixation (A + P: SynCage with transpedicular fixation). The ROM comparison of the instrumented models with normal and osteoporotic bone was normalized by the corresponding value of theintactmodel.

\section{Convergence and validation of the intact model}

The convergence test consisted of three mesh qualities: 4,750 elements / 4,960 nodes for the coarse model, 27,244 elements / 30,630 nodes for normal model, and 84,594 elements / 94,162 nodes for the finest model. For the finest mesh quality, the changes in total ROM were respectively within $1.03 \%$ in flexion $\left(<0.2^{\circ}\right), 4.39 \%$ in extension $\left(<0.5^{\circ}\right), 0.01 \%$ in torsion $\left(<0.2^{\circ}\right)$, and $0.001 \%$ in lateral bending $\left(<0.1^{\circ}\right)$. Consequently, this study used the finest model to evaluate the biomechanical behaviors of the INT and ALIF models under four physiological motions.

For model validation, the ROM changes in five levels of the INT model were compared with the experimental results of Rohlmann's study [27]. Under 3.75and $7.5-\mathrm{Nm}$ moments with $150-\mathrm{N}$ preload, a previous study [31] by the current authors showed data of the current INT model within the extreme values of Rohlmann's results. Under a $10-\mathrm{Nm}$ moment with $150 \mathrm{~N}$ preload, however, the predicted ROMs of this study were $6^{\circ}$ to $11^{\circ}$ less than those of the in vitro tests 


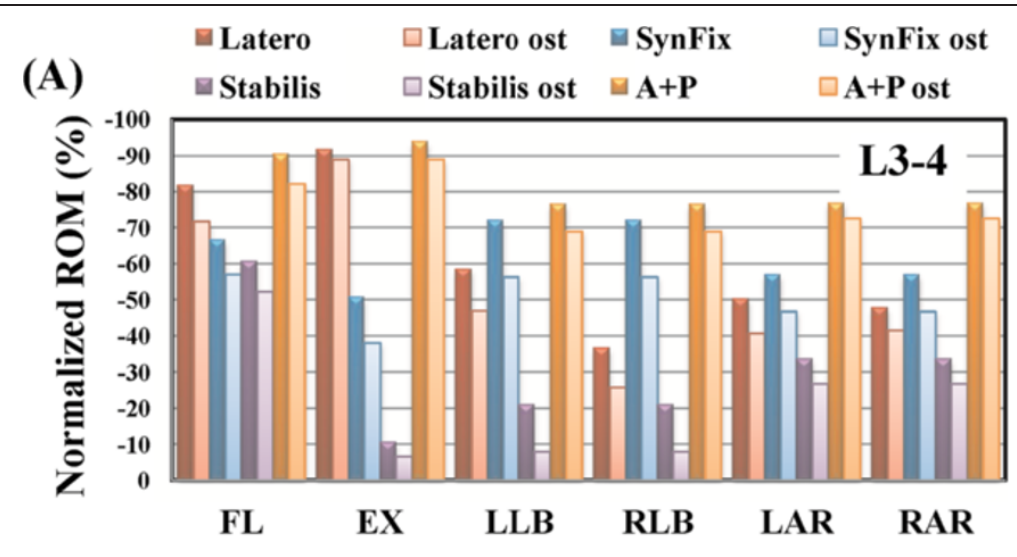

(B)

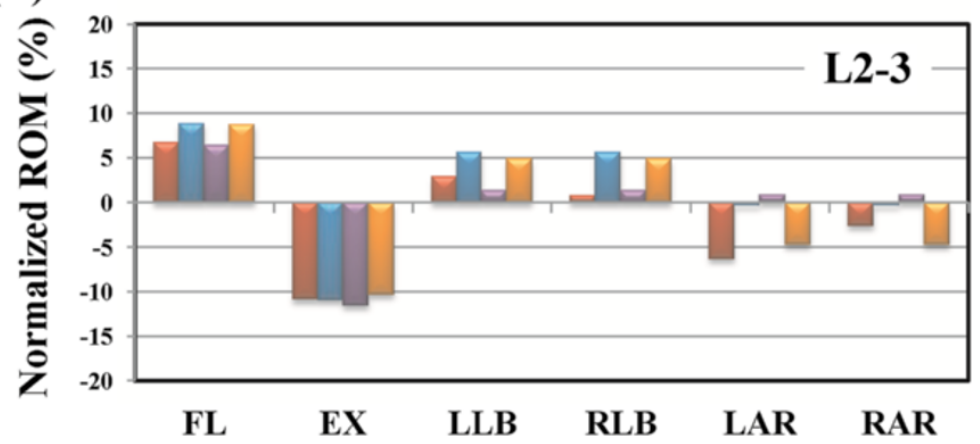

(C)

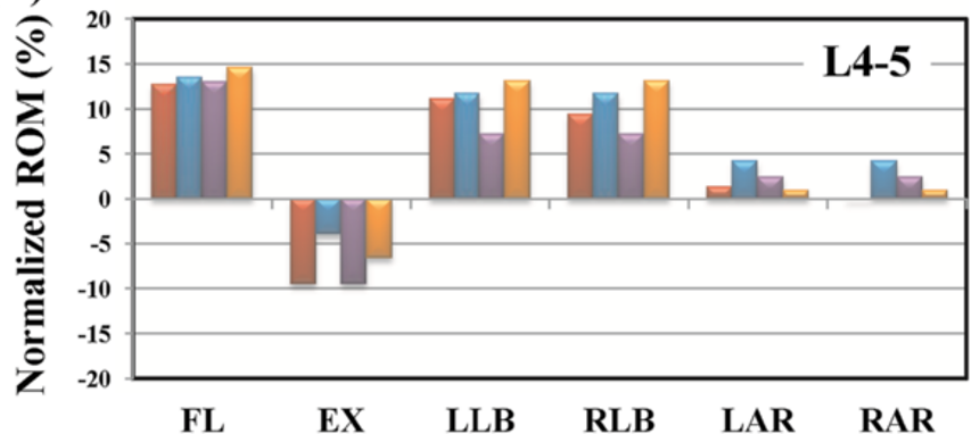

Figure 3 Comparison of the normalized intersegmental ROM among all models under six motions. (A) Surgical level. (B-C) Adjacent levels.

under flexion. This might be explained by the differing preload applications of the current (pressure preload) and earlier in vitro tests (vertical preload) $[12,14]$. The pressure preload applied a compressive force of $150 \mathrm{~N}$ that was always perpendicular to the lumbar top. However, the compression of the vertical preload was consistently orthogonal to the horizontal plane during lumbar motion. Consequently, the pressure preload resulted in a much lower bending moment compared with the vertical preload. In torsion, the facet contact force of the INT model ranged between 121 to $130 \mathrm{~N}$ and the values were within the ranges of earlier studies [25,32]. This indicated that the INT model was well verified for further simulation of the four ALIF models. In total, there were nine models (one intact, four instrumented $\times$ two bones) and six motions simulated in this study.

Intersegmental ROM at the surgical and adjacent levels Referring to Panjabi's ALE formula [33], the restricted ROM of the instrumented model was compared with the corresponding ROM value of the INT model (Figure 3).

The $\mathrm{A}+\mathrm{P}$ model had the maximal capability in restricting $\mathrm{ROM}$ from $-76.5 \%$ to $-93.8 \%$ in all motions. The Latero model performed ROM control similar to that of $\mathrm{A}+\mathrm{P}$, and superior to that of the SynFix model in flexion and extension. Moreover, the Latero model 


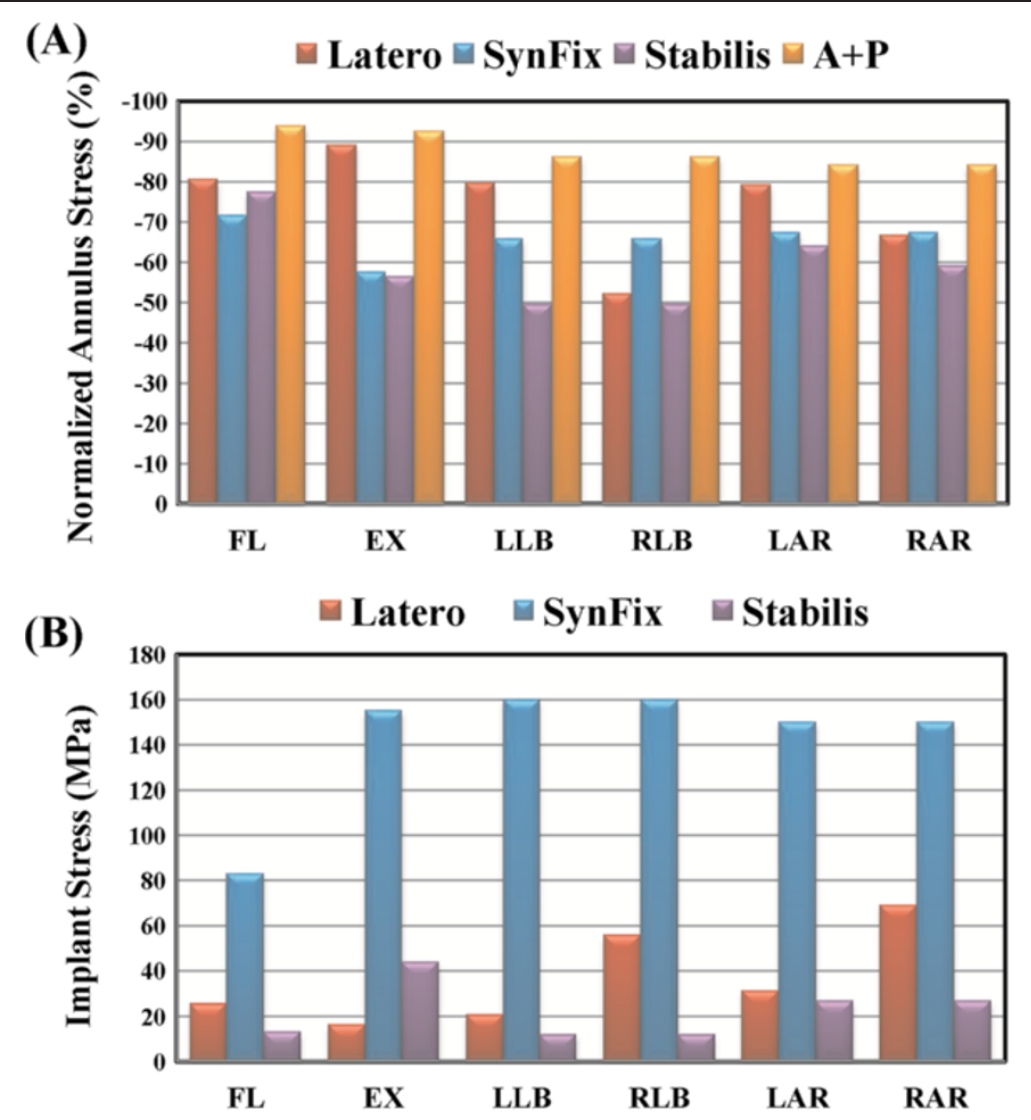

Figure 4 Stress comparison of the normalized stress among all models under six motions. (A) Annulus stress. (B) Implant stress.

performance was similar to that of the SynFix model in bilateral axial rotation and left lateral bending, but inferior in right lateral bending. The asymmetrical design of the Latero model with the vertebral plate placed on the left side explained the different behaviors in left versus right lateral bending. The Stabilis model had the lowest values in all motions, especially in controlling lateral bending $(-21.0 \%)$ and extension $(-10.6 \%)$. This finding is consistent with the result of the previous study by the current authors [14].

For the osteoporotic lumbar, the percentages of restricted ROM at the surgical level for all models were also shown in Figure 3. Under the osteoporotic condition, the percentages of restricted ROM consistently decreased for all models. The percentages of change rate were $2.7 \% \sim 10.8 \%$ in the Latero, $9.7 \% \sim 15.9 \%$ in the SynFix, $4.4 \% \sim 13.0 \%$ in the Stabilis, and $4.8 \% \sim 8.2 \%$ in the $\mathrm{A}+\mathrm{P}$ models. The maximal change rate was around $10 \%$ in the Latero (10.8\%) and A + P (8.2\%) models, and slightly higher in the SynFix (15.9\%) and Stabilis (13.0\%) models. Under the osteoporotic condition, the $\mathrm{A}+\mathrm{P}$ model still had $70 \%$ of ROM control; while the Latero model had weaker control in right lateral bending $(-25.9 \%$, versus $-36.7 \%$ in normal bone), the SynFix model had weaker control in extension $(-38.0 \%$, versus $-50.7 \%$ in normal bone), and the Stabilis model had even weaker control in extension (-6.2\%) and lateral bending (-8.0\%).

ROM control of the adjacent levels under normal and osteoporosis conditions for all models were shown in Figures 3a-c. Under both conditions, the percentages of ROM change rate were less than $12.3 \%$ at L2-3, and less than $15.8 \%$ at the L4-5 level as compared to the INT model. The difference of ROM change rates was small at the adjacent levels of each model under normal and osteoporotic conditions.

\section{Annulus stress}

Referring to Panjabi's formula [33], the normalized percentages of the maximum annulus stress at the surgical level of all models were shown in Figure 4a. At the surgical level, the normalized annulus stress and intersegmental ROM can be well correlated for each model. The Latero model had maximum annulus stress similar to the A + P model, and was superior to the SynFix model in flexion and extension. It was also similar to the SynFix model in bilateral axial rotation and left lateral bending, but inferior in right lateral bending. 


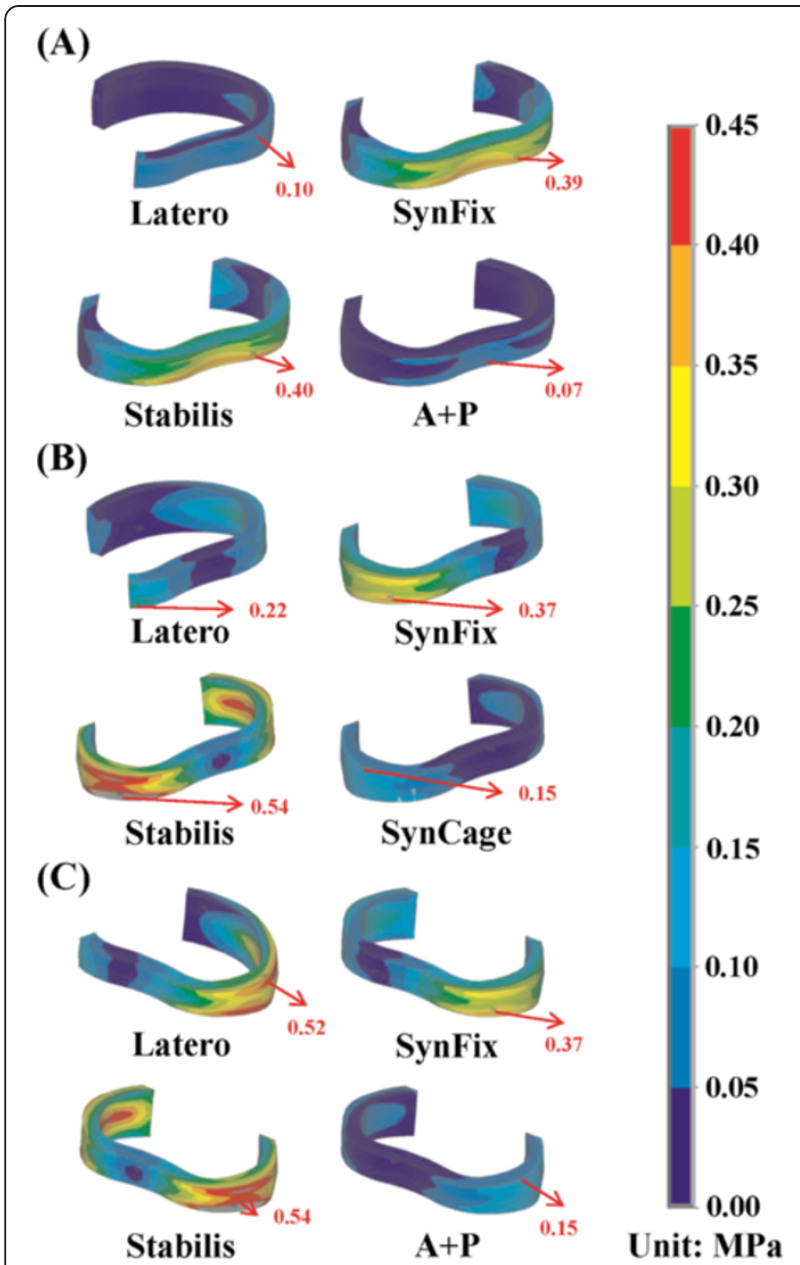

Figure 5 Stress distribution of the ground substance at the surgical level for all models. (A) Extension (B) Left lateral bending. (C) Right lateral bending.

Under the normal condition, the annulus stress distribution of the four instrumented models was shown in extension, right and left lateral bending (Figures 5a-c). The Latero had annulus stress distribution similar to the A + P model in extension (Figure 5a), similar to the Stabilis model in right lateral bending (Figure $5 \mathrm{~b}$ ), and similar to the SynFix model in left lateral bending (Figure $5 \mathrm{c}$ ). The Stabilis model showed the highest annulus stress in all motions, with the stress being concentrated at the posterior annulus in extension (Figure $5 \mathrm{a}$ ) and at the lateral annulus in bilateral lateral bending (Figures $5 \mathrm{~b}, \mathrm{c}$ ). In contrast, the annulus stress was more evenly distributed in the other three models. However, at the adjacent L2-3 and L4-5 levels, there was no obvious difference in annulus stress distribution among the four instrumented models.

\section{Facet contact force}

The facet contact forces at the surgical and adjacent levels of all models are shown in extension and bilateral rotation (Figures $6 \mathrm{a}-\mathrm{c}$ ). The $\mathrm{A}+\mathrm{P}$ model had nearly zero facet contact force at the surgical level in all motions, because the relative motions of the facets joint were restricted by the pedicle screw. In extension, the Latero model could control most of extension (-91.6\%) and there was little force shifted to the facet joints (Figure 6a). In bilateral axial rotation, the facet contact force of the Latero and SynFix models were similar, because both devices had similar capability in controlling axial rotation (Figures 6b-c). The highest values of facet contact force at the surgical level of the Stabilis model could be explained by the poorer control of extension and axial rotation. At the adjacent levels, there were small differences $(<15.0 \mathrm{~N})$ of facet contact force among the four models in extension and axial rotation (Figures 6a-c; left: left facet and right: right facet).

\section{Implant stress}

The maximum stresses sustained by the integrated parts of three stand-alone cages were shown in Figure $4 \mathrm{~b}$. The locking screws of the SynFix model had higher stress than the vertebral plate of Latero and the threaded cylinder of Stabilis models in all motions. The ratios of maximum stresses at the integrated parts among Latero, SynFix and Stabilis models were 1: 9.6: 2.7 in extension, 1: 2.9: 0.2 in right lateral bending, and $1: 2.2$ : 0.4 in right axial rotation, respectively. The maximum stress at the integrated parts of the Latero model was similar to that of the Stabilis model in left lateral bending and left axial rotation.

The stress distribution at the integrated part of the three stand-alone models was shown in extension, right lateral bending, and right axial rotation (Figures $7 \mathrm{a}-\mathrm{c}$ ). In these three motions, the stress was concentrated at the base of the locking screws in the SynFix model; while the stress was evenly distributed over the vertebral plate in the Latero model and the threaded cylinder in the Stabilis model.

\section{Endplate stress}

Since cage subsidence most commonly occurs at the lower endplate of the upper vertebra [2], only the maximum stress on the L3 endplate was calculated. All models consistently showed that the maximum stress occurs at the top surface of the L3 vertebra (Figure 8). The adjacent endplate of the Latero model was more stressed than the other models in axial rotation. Among all motions, the L3 endplate was most stressed in flexion at the SynFix model. In extension, the stress ratios of the contact surface on L3 between the three stand-alone cage models in extension equal to 1:1.07:1.15 in Latero, SynFix, and Stabilis, respectively. The aforementioned stress ratios were 1:1.47:0.53, 1:0.48:0.53 and 1:0.31:0.34 in flexion, left axial rotation and right axial rotation, respectively. The highly stressed 


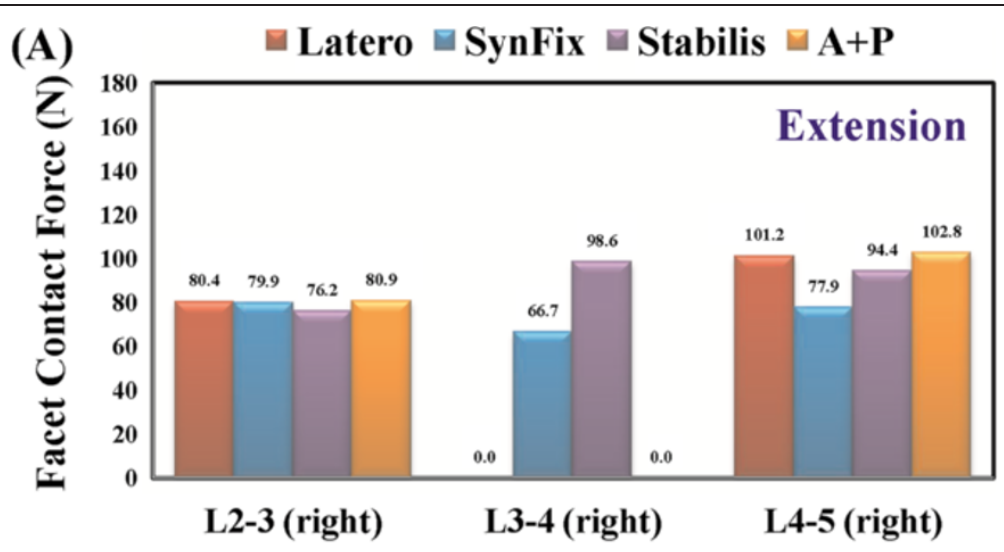

(B)

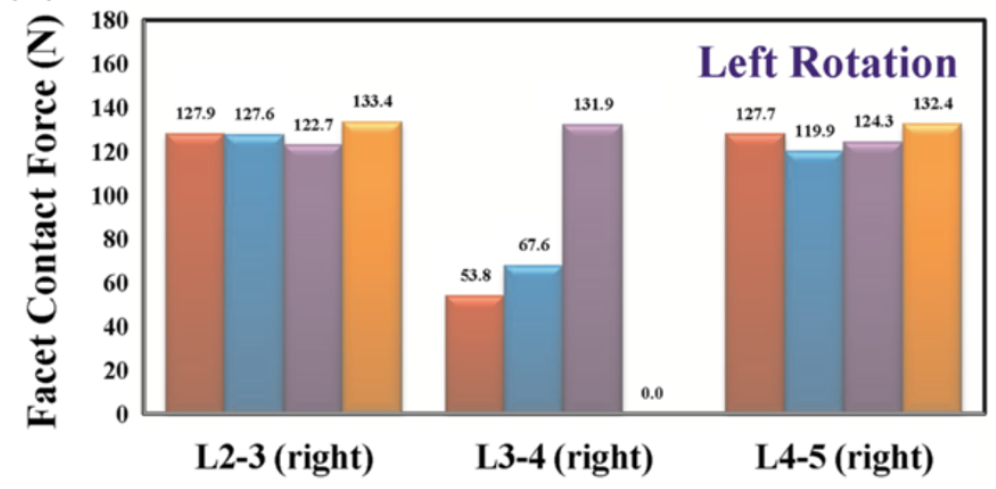

(C)

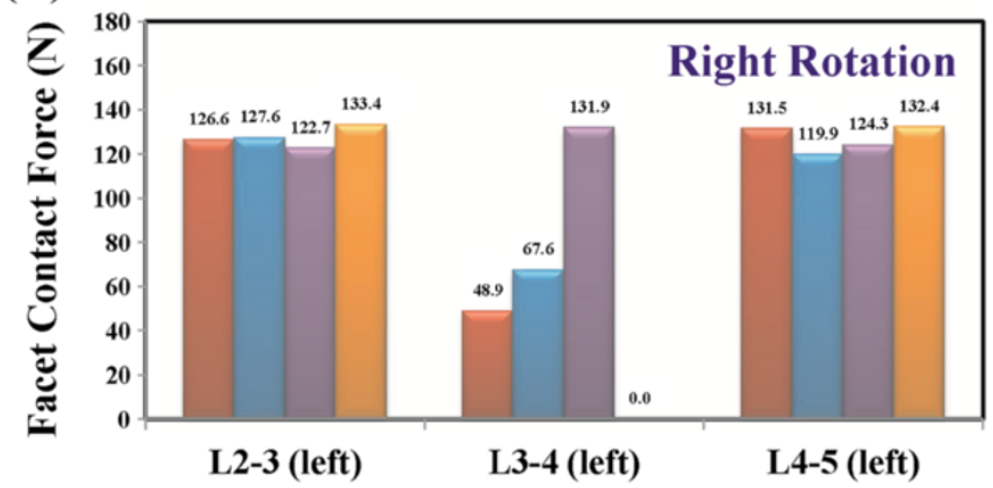

Figure 6 Comparison of facet contact force among all models. (A) Extension. (B) Left axial rotation. (C) Right axial rotation. Middle bars are the surgical level ( $L 3-4)$; left and right bars are the adjacent levels ( $L 2-3$ and $L 4-5$ ).

endplate of the Latero model in axial rotation might be attributed to the smaller contact surface of the Latero cage.

\section{Discussion}

Nowadays, the interbody fusion cage with transpedicular fixation has been established as the standard for lumbar degenerative disorders due to its high fusion rate. However, there have been concerns about approach- and device-related issues as well as adjacent segment disease (ASD). ASD is an important long-term issue in lumbar fusion. The literature has shown the incidence of ASD when using pedicle screws is much higher than that for ALIF, where the incidence of ASD after anterior fusion is similar to that under natural conditions [34]. Recently, surgical trends have shifted using minimally invasive techniques through alternative approaches and reduced loads of fixation devices to mitigate the issues of concern. Examples of such techniques are anterior standalone cages coupled with self-stabilizing mechanisms, such as the Latero plate, SynFix screw, and Stabilis 

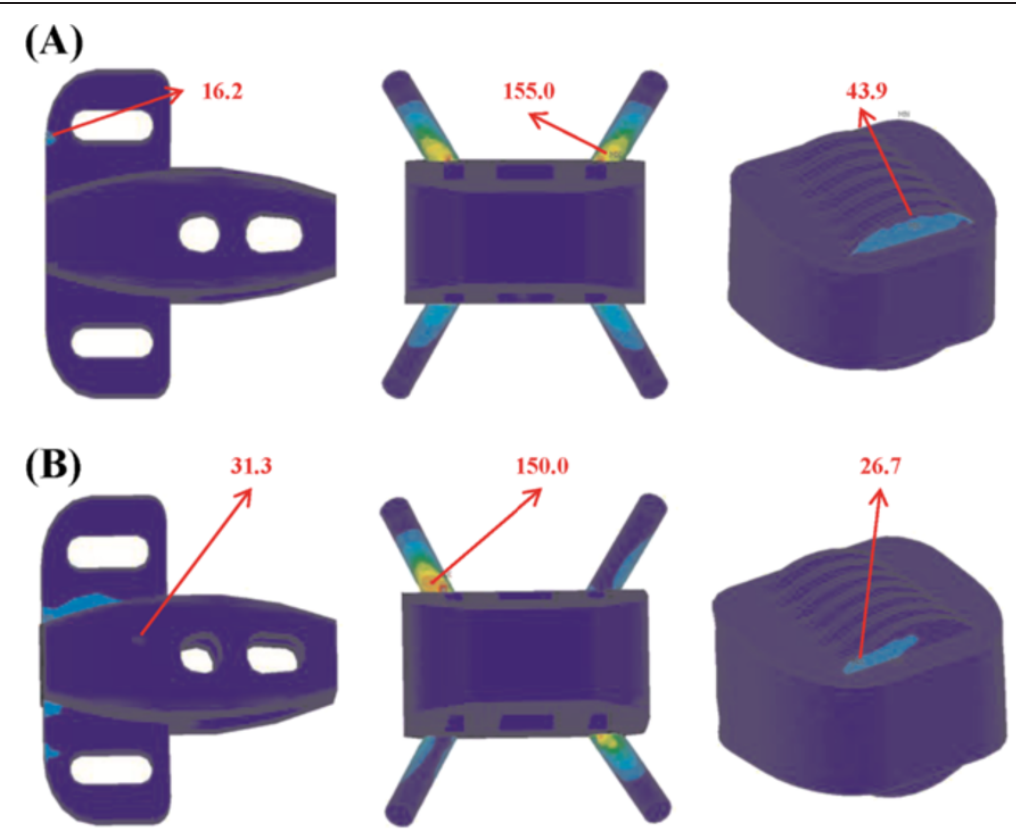

\section{(C)}
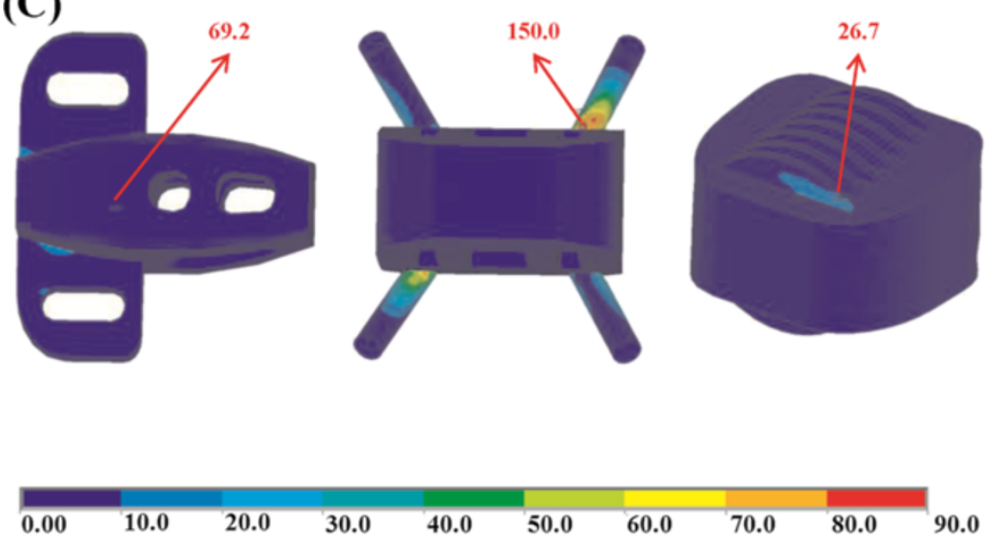

Unit: MPa

Figure 7 Distribution of implant stress for the Latero, SynFix, and Stabilis models. (A) Extension. (B) Left lateral bending. (C) Right lateral bending.

cylinder, which eliminate the need of posterior fixation (Figure 1). Despite the claimed improvements in construct stability, anterior access has disadvantages, including risk of vascular injury and sacrificing of the anterior longitudinal ligament [35].

In this study, the results of ROM control showed that the $\mathrm{A}+\mathrm{P}$ model had the best overall performance and the Stabilis model had the weakest ROM control. Both Latero and SynFix models were shown to have $>50 \%$ of ROM control in all motions except for that of the Latero model, which demonstrated a discrepant control of left $(-59 \%)$ and right lateral bending $(-37 \%)$. The asymmetrical design of the Latero cage and its instrumentation in the left side of interspace may explain the different left and right bending behaviors. In practice, this discrepancy might be compensated for by adding bone grafts or elongating the cage to cover the right side of the interspace. Various biomechanical studies have shown that stand-alone cages lack control of extension and axial rotation but not of flexion and lateral bending [7]. The Latero model $(-92 \%)$ had comparable control in extension as the A + P model (-94\%) and was significantly superior to the SynFix model $(-51 \%)$. This could be explained by the force vector of extension which was directly perpendicular to the Latero plate. This also accounts for the excellent control of extension and axial rotation by the Latero plate in comparison to the other counterparts (Figure 3).

Under osteoporotic conditions, all models lost a little ROM control at the surgical level, ranging from 3\% to 
(A)

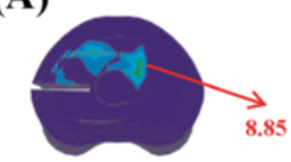

Latero

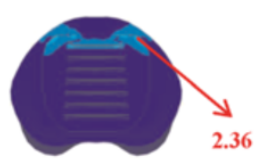

Stabilis

(C)

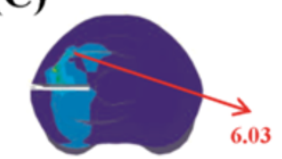

Latero

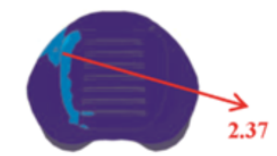

Stabilis

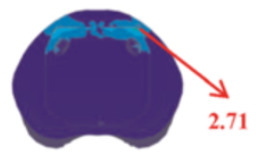

SynFix

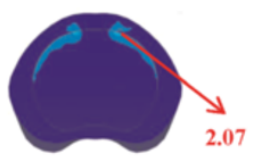

$\mathbf{A}+\mathbf{P}$

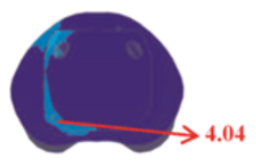

SynFix

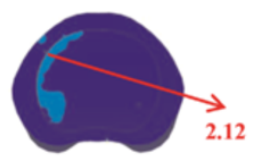

$\mathbf{A}+\mathbf{P}$

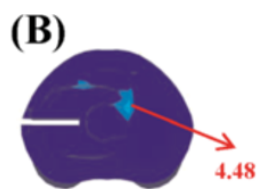

Latero

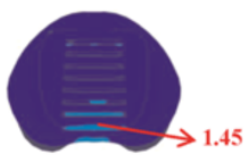

Stabilis

(D)

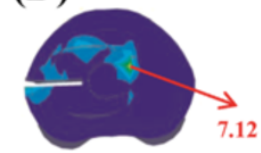

Latero

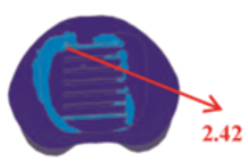

Stabilis

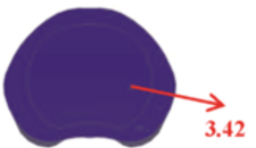

SynFix

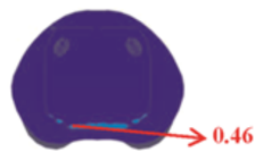

$\mathbf{A}+\mathbf{P}$

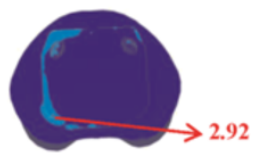

SynFix

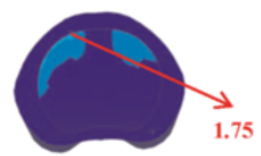

$\mathbf{A}+\mathbf{P}$

\section{$\begin{array}{llllllllll}0.00 & 0.70 & 1.40 & 2.10 & 2.80 & 3.50 & 4.20 & 4.90 & 5.60 & 6.30\end{array}$}

\section{Unit: MPa}

Figure 8 Distribution of endplate stress on the upper surface of the L4 vertebra for all models. (A) Flexion. (B) Extension. (C) Left lateral bending. (D) Left axial rotation.

$16 \%$ (Figure 3). In real life with osteoporosis, the screwbased devices (SynFix and $\mathrm{A}+\mathrm{P}$ ) potentially exhibit screw loosening after cyclic loading. Although the rigidity of the screw-bone interface may be enhanced by injecting cement, there is the possibility of cement-related hazards. In Stabilis, the bony endplates might be compromised by threading in the cylindrical cage, and a higher incidence of cage subsidence than in other cages has been reported [2]. The Latero plate utilizes a stabilizing mechanism which is dissimilar from those of other devices; it remains to be proved clinically whether the Latero model behaves differently in an osteoporotic lumbar. At the adjacent levels, their segmental ROMs were indeed changed when compared with the intact state. However, the changes were small among these four models, even in the stiffest A + P model which is known to have a higher incidence of ASD at long-term follow up. This finite-element study cannot reflect the consequences of clinical cyclic loading, it can only reveal the ROM changes of adjacent levels.

The varying design concepts of the integrated parts in the self-stabilizing cages not only affect their ROM control, but also influence the stress distribution on the device and the adjacent tissues. In this study, the stress distribution on the Latero plate was much lower and more evenly distributed than that of the SynFix screws (Figure 7). The SynFix screws sustained 3.75 to 9.57 times greater stresses than the Latero plate, with the stress concentrated at the cage-screw junctions. For the Latero and SynFix models, the difference in modes and amounts of distributed stress might be explained by the different configurations of the stabilizing components: sizes, contact areas, and the distance to the center of rotation. In cyclic loading, the Latero plate might be less likely to have fatigue failure than the SynFix screws. The cylindrical cage of the Stabilis model sustained the lowest stress as a result of its relatively suboptimal ROM control.

Stress distribution on the vertebra-cage interface represents vertical compression force exerted on the bony endplate. The Latero model exerted the highest stress on the L4 endplate compared to the other models (Figure 8). This might be due to the stress not being fully shielded by the intervertebral plate and being redistributed by the plate and transmitted onto the L4 endplate. Comparatively, the stress was shielded by the screws of the SynFix and $\mathrm{A}+\mathrm{P}$ models and thus less stress was distributed on the endplate. In the Stabilis model, because of its relatively poor ROM control, the distributed stress on the 
endplate was less than in the other three models. There were two biomechanical implications in the Latero model, which had higher vertical load on the L4 endplate than the other counterparts. The first implication indicates that higher vertebral stress may increase the incidence of cage subsidence particularly in suboptimal bone density. On the other hand, unshielded vertical load may be beneficial for graft consolidation according to Wolff's law.

After interbody fusion, abnormally high transmission of loads to the facet joints may ultimately result in arthritic changes. At the surgical level, when comparing the three stand-alone cage models, the Latero model was shown to have the lowest values of facet contact force in bilateral rotation and was near absent in extension (Figure 6). The facet joints at the surgical level seemed to be relatively well protected in the Latero model in comparison to the SynFix and Stabilis models. At the adjacent levels, there were few differences of facet contact force among all four instrumented models. This indicates that the reasons for the degenerative facet joints at the adjacent levels might be attributed to the other clinical factors.

Distribution of annulus stress can provide the clinical implication that higher stress may result in annulus disruption and disc herniation [28-30]. At the surgical level for the four instrumented models, the distribution of annulus stress was well correlated inversely to their ROM control (Figures 3, 4a). At right lateral bending, the highest annulus stress was found in the Latero model, where it was concentrated at the right annulus. This corresponded to the relatively inferior control of the Latero due to its asymmetrical design. The posterior and left sides of annulus in the Stabilis model sustained the highest stress at extension and lateral bending, which manifested in its inferior control of those moments.

\section{Conclusions}

There were several limitations inherent in this study. Bone fusion was not included and only the effect of the initial stability was considered for the stand-alone cages. Degenerative discs are common in most patients with ALIF surgery; however, it is challenging in modeling to assign material properties to various grades of degenerative discs, such as delamination, dehydration, or reduced disc height. These variations of the lumbar tissues were not included in this study. In addition, the geometry of implants was somewhat simplified for mesh modeling. This study was not concerned with the effect of bone ingrowth into the cage and ligament pretension after inserting the implants. The stabilizing behaviors of trunk muscles were mimicked by the follower load that has been extensively used in related in vitro tests [16,27]. However, the real situations of muscle contraction and complicated external load conditions in vivo were not investigated. The fatigue failure of the cages' components was not studied in the static simulation and the current authors we recommend that they should be evaluated by experimental or clinical observation.

In conclusion, this study extensively compared the stabilizing mechanisms of three stand-alone ALIF cages. The vertebral plate of the Latero model provided sufficient ability in stabilizing intersegmental motions: it was comparable to the A + P model in flexion and extension, comparable to the SynFix in bilateral axial rotation and left lateral bending, and inferior but compensable in right lateral bending. In contrast, the Stabilis model was less favorable due to its poorer control in extension and lateral bending. Further experimental and clinical studies should be conducted to validate the numerical observations.

\section{Abbreviations}

ALIF: Anterior lumbar interbody fusion; INT: Intact model; A + P: Model, SynCage with transpedicular fixation; ROM: Range of motion; FL: Flexion; EX: Extension; LLB: Left lateral bending; RLB: Right lateral bending; LAR: Left axial rotation; RAR: Right lateral bending.

\section{Competing interests}

The authors declare that they have no competing interests.

\section{Authors' contributions}

MCC participated in the study design, in collecting the data, the statistically analyses and drafting of the manuscript. SHC, JFL and $\mathrm{CHH}$ participated in the study design. SCL advised and assisted drafting of the manuscript. All authors read and approved the final manuscript.

\section{Acknowledgements}

This study was supported by a grant from the National Science Council of the Republic of China. The computing facilities provided by the National Center for High-Performance Computing are greatly appreciated.

\section{Author details}

'Department of Orthopedics, Tzu-Chi General Hospital at Taichung and Tzu Chi University, Taichung, Taiwan. '2Department of Mechanical Engineering, National Chiao Tung University, 1001 University Road, Hsinchu 30010, Taiwan. ${ }^{3}$ BoneCare Orthopedic Centers, Han-Chiung Clinics, Taipei, Taiwan. ${ }^{4}$ Graduate Institute of Biomedical Engineering, National Taiwan University of Science and Technology, Taipei, Taiwan.

Received: 6 March 2013 Accepted: 24 September 2013 Published: 2 October 2013

\section{References}

1. Schleicher P, Gerlach R, Schár B, Cain CM, Achatz W, Pflugmacher R, Haas NP, Kandziora F: Biomechanical comparison of two different concepts for stand alone anterior lumbar interbody fusion. Eur Spine J 2008, 17:1757-1765

2. Cho CB, Ryu KS, Park CK: Anterior lumbar interbody fusion with standalone interbody cage in treatment of lumbar intervertebral foraminal stenosis: comparative study of two different types of cages. J Korean Neurosurg Soc 2010, 47:352-357.

3. Brantigan JW, Steffee AD, Lewis ML, Quinn LM, Persenaire JM: Lumbar interbody fusion using the Brantigan I/F cage for posterior lumbar interbody fusion and the variable pedicle screw placement system: two-year results from a Food and Drug Administration investigational device exemption clinical trial. Spine 2000, 25:1437-1446.

4. Kuslich SD, Danielson G, Dowdle JD, Sherman J, Fredrickson B, Yuan H, Griffith SL: Four-year follow-up results of lumbar spine arthrodesis using the Bagby and Kuslich lumbar fusion cage. Spine 2000, 25:2656-2662.

5. Pavlov PW, Spruit M, Havinga M, Anderson PG, van Limbeek J, Jacobs WC: Anterior lumbar interbody fusion with threaded fusion cages and autologous bone grafts. Eur Spine J 2000, 9:224-229. 
6. Steffen T, Tsantrizos A, Aebi M: Effect of implant design and endplate preparation on the compressive strength of interbody fusion constructs. Spine 2000, 25:1077-1084.

7. Oxland TR, Lund T: Biomechanics of stand-alone cages and cages in combination with posterior fixation: a literature review. Eur Spine J 2000 9:S95-S101.

8. Steffen T, Tsantrizos A, Fruth I, Aebi M: Cage: designs and concepts. Eur Spine J 2000, 9:S89-S94.

9. Costa F, Sassi M, Ortolina A, Cardia A, Assietti R, Zerbi A, Lorenzetti M, Galbusera F, Fornari M: Stand-alone cage for posterior lumbar interbody fusion in the treatment of high-degree degenerative disc disease: design of a new device for an "old" technique. A prospective study on a series of 116 patients. Eur Spine J 2011, 20:S46-S56.

10. Tsuang YH, Chiang YF, Hung CY, Wei HW, Huang CH, Cheng CK. Comparison of cage application modality in posterior lumbar interbody fusion with posterior instrumentation-A finite element study. Med Eng Phys 2009, 31:565-570.

11. Lin RM, Huang KY, Lai KA: Mini-open anterior spine surgery for anterior lumbar diseases. Eur Spine J 2008, 17:691-697.

12. Cain CM, Schleicher P, Gerlach R, Pflugmacher R, Scholz M, Kandziora F: A new stand-alone anterior lumbar interbody fusion device: biomechanical comparison with established fixation techniques. Spine 2005, 30(23):2631-2636.

13. Kim Y: Finite element analysis of anterior lumbar interbody fusion: threaded cylindrical cage and pedicle screw fixation. Spine 2007 32:2558-2568

14. Chen SH, Tai CL, Lin CY, Hsieh PH, Chen WP: Biomechanical comparison of a new stand-alone anterior lumbar interbody fusion cage with established fixation techniques - a three-dimensional finite element analysis. BMC Musculoskelet Disord 2008, 9:88.

15. Jost B, Cripton PA, Lund T, Oxland TR, Lippuner $K$, Jaeger $P$, Nolte LP: Compressive strength of interbody cages in the lumbar spine: the effect of cage shape, posterior instrumentation and bone density. Eur Spine J 1998, 7:132-141.

16. Patwardhan AG, Havey RM, Meade KP, Lee B, Dunlap B: A follower load increases the load-carrying capacity of the lumbar spine in compression. Spine 1999, 24:1003-1009.

17. Kettler A, Wilke HJ, Dietl R, Krammer M, Lumenta C, Claes L: Stabilizing effect of posterior lumbar interbody fusion cages before and after cyclic loading. J Neurosurg 2000, 92:87-92.

18. Kim Y: Prediction of mechanical behaviors at interfaces between bone and two interbody cages of lumbar spine segments. Spine 2001, 26:1437-1442

19. Oxland TR, Lund $T$, Jost $B$, Cripton $P$, Lippuner $K$, Jaeger $P$, Nolte LP: The relative importance of vertebral bone density and disc degeneration in spinal flexibility and interbody implant performance. Spine 1996, 21:2558-2569.

20. Polikeit A, Ferguson SJ, Nolte LP, Orr TE: Factors influencing stresses in the lumbar spine after the insertion of intervertebral cages: finite element analysis. Eur Spine J 2003, 12:413-420.

21. Silva MJ, Keaveny TM, Hayes WC: Load sharing between the shell and centrum in the lumbar vertebral body. Spine 1997, 22:140-150.

22. Lu YM, Hutton WC, Gharpuray VM: Do bending, twisting, and diurnal fluid changes in the disc affect the propensity to prolapse? A viscoelastic finite element model. Spine Nov 1996, 21(22):2570-2579.

23. Schmidt H, Heuer F, Simon U, Kettler A, Rohlmann A, Claes L, Wilke HJ: Application of a new calibration method for a three-dimensional finite element model of a human lumbar annulus fibrosus. Clin Biomech (Bristol, Avon) May 2006, 21(4):337-344.

24. Carter DR, Hayes WC: The compressive behavior of bone as a two-phase porous structure. J Bone Joint Surg Am 1977, 59(7):954-62.

25. Goel VK, Monroe BT, Gilbertson LG, Brinckmann P: Interlaminar shear stresses and laminae separation in a disc. Finite element analysis of the L3-L4 motion segment subjected to axial compressive loads. Spine 1995 20:689-698.

26. Renner SM, Natarajan RN, Patwardhan AG, Havey RM, Voronov LI, Guo BY, Andersson GB, An HS: Novel model to analyze the effect of a large compressive follower pre-load on range of motions in a lumbar spine. J Biomech 2007, 40:1326-1332.
27. Rohlmann A, Neller S, Claes L, Bergmann G, Wilke HJ: Influence of a follower load on intradiscal pressure and intersegmental rotation of the lumbar spine. Spine 2001, 26:E557-E561.

28. Edwards WT, Ordway NR, Zheng Y, McCullen G, Han Z, Yuan HA: Peak stresses observed in the posterior lateral anulus. Spine 2001, 26:1753-1759.

29. Adams MA, Hutton WC: The mechanics of prolapsed intervertebral disc. Int Orthopaed 1982, 6:249-253.

30. McNally DS, Adams MA, Goodship AE: Can intervertebral disc prolapse be predicted by disc mechanics? Spine 1993, 18:1525-1530.

31. Chen SH, Zhong ZC, Chen CS, Chen WJ, Hung C: Biomechanical comparison between lumbar disc arthroplasty and fusion. Med Eng Phys 2009, 31:244-253

32. Goel VK, Kong W, Han JS, Weinstein JN, Gilbertson LG: A combined finite element and optimization investigation of lumbar spine mechanics with and without muscles. Spine 1993, 18:1531-1541.

33. Panjabi MM: Hybrid multidirectional test method to evaluate spinal adjacent-level effects. Clin Biomech 2007, 22:257-265.

34. Bae JS, Lee SH, Kim JS, Jung B, Choi G: Adjacent segment degeneration after lumbar interbody fusion with percutaneous pedicle screw fixation for adult low-grade isthmic spondylolisthesis: minimum 3 years of follow-up. Neurosurg. 2010, 67:1600-1607.

35. Ploumis A, Wu C, Fischer G, Mehbod AA, Wu W, Faundez A, Transfeldt EE: Biomechanical comparison of anterior lumbar interbody fusion and transforaminal lumbar interbody fusion. J Spinal Disord Tech 2008, 21:120-125.

doi:10.1186/1471-2474-14-281

Cite this article as: Chen et al.: Biomechanical comparison of three stand-alone lumbar cages - a three-dimensional finite element analysis. BMC Musculoskeletal Disorders 2013 14:281.

\section{Submit your next manuscript to BioMed Central and take full advantage of:}

- Convenient online submission

- Thorough peer review

- No space constraints or color figure charges

- Immediate publication on acceptance

- Inclusion in PubMed, CAS, Scopus and Google Scholar

- Research which is freely available for redistribution 